\title{
Development and Validation of 11 Symptom Indexes to Evaluate Response to Chemotherapy for Advanced Cancer
}

\author{
David Cella, PhD ${ }^{\mathrm{a}, \mathrm{b}, \mathrm{c}}$; Sarah K. Rosenbloom, $\mathrm{PhD}^{\mathrm{a}, \mathrm{b}}$; Jennifer L. Beaumont, MS ${ }^{\mathrm{a}}$; Susan E. Yount, $\mathrm{PhD}^{\mathrm{a}, \mathrm{c}}$; \\ Diane Paul, MS, RN ; Debra Hampton ${ }^{\text {d; }}$ Amy P. Abernethy, MD ${ }^{\mathrm{e}}$; Paul B. Jacobsen, PhD; \\ Karen Syrjala, $\mathrm{PhD}^{\mathrm{s}}$; and Jamie H. Von Roenn, $\mathrm{MD}^{\mathrm{h}}$; Chicago, Illinois; Fort Washington, Pennsylvania; \\ Durham, North Carolina; Tampa, Florida; and Seattle, Washington
}

\section{Key Words}

Symptom index, measurement, advanced cancer, patientcentered, health-related quality of life

\begin{abstract}
Recent guidance from the FDA discusses patient-reported outcomes as end points in clinical trials. Using methods consistent with this guidance, the authors developed symptom indexes for patients with advanced cancer. Input on the most important symptoms was obtained from 533 patients recruited from NCCN Member Institutions and 4 nonprofit social service organizations. Diagnoses included bladder, brain, breast, colorectal, head and neck, hepatobiliary/pancreatic, kidney, lung, ovarian, and prostate cancers and lymphoma. Physician experts in each of these
\end{abstract}

From the a Department of Medical Social Sciences, ${ }^{b}$ Department of Psychiatry and Behavioral Sciences, and Institute for Healthcare Studies, Northwestern University Feinberg School of Medicine,

Chicago, Illinois; 'Oncology Research Program, National

Comprehensive Cancer Network, Fort Washington, Pennsylvania;

eDivision of Medical Oncology, Department of Medicine, Duke

University Medical Center, Durham, North Carolina; ${ }^{f}$ Health

Outcomes and Behavior Program, H. Lee Moffitt Cancer Center,

Tampa, Florida; 'Biobehavioral Sciences, Clinical Research Division,

Fred Hutchinson Cancer Research Center, and Department of

Psychiatry and Behavioral Sciences, University of Washington

School of Medicine, Seattle, Washington; and hDivision of

Hematology/Oncology, Robert H. Lurie Comprehensive Cancer

Center, Northwestern University Feinberg School of Medicine,

Chicago, Illinois.

Submitted December 17, 2009; accepted for publication

May 18, 2010.

The authors have disclosed that they have no financial interests, arrangements, or affiliations with the manufacturers of any products discussed in this article or their competitors. Support for the study was provided by grants from the following

pharmaceutical companies: Amgen, AstraZeneca, Bayer, Bristol-

Myers Squibb, Centocor, Cell Therapeutics, Inc., Eli Lilly and

Company, Genentech, GlaxoSmithKline, Merck \& Co., Novartis,

Ortho Biotech, Pfizer, sanofi-aventis, and Takeda Pharmaceuticals.

Correspondence: David Cella, PhD, Robert H. Lurie Comprehensive

Cancer Center, Department of Medical Social Sciences,

Northwestern University, $710 \mathrm{~N}$ Lake Shore Drive - 7th Floor,

Chicago, IL 60611. E-mail: d-cella@northwestern.edu diseases were also surveyed to differentiate symptoms that were predominantly disease-based from those that were predominantly treatment-induced. Results are evaluated alongside previously published indexes for 9 of these 11 advanced cancers that were created based on expert provider surveys, also implemented at NCCN Member Institutions. Final results are 11 symptom indexes that reflect the highest priorities of people affected by these 11 advanced cancers and the experienced perspective of the people who provide their medical treatment. Beyond the clinical value of such indexes, they may also contribute significantly to satisfying regulatory requirements for a standardized tool to evaluate drug efficacy with respect to symptomatology. (JNCCN 2011;9:268-278)

\section{Overview}

Recent guidance from the FDA discusses patientreported outcomes as end points in clinical trials. Using methods consistent with this guidance, the authors developed symptom indexes for patients with advanced cancer. Input on the most important symptoms was obtained from 533 patients recruited from NCCN Member Institutions and 4 nonprofit social service organizations. Diagnoses included bladder, brain, breast, colorectal, head and neck, hepatobiliary/pancreatic, kidney, lung, ovarian, and prostate cancers and lymphoma. Physician experts in each of these diseases were also surveyed to differentiate symptoms that were predominantly disease-based from those that were predominantly treatment-induced. Results are evaluated alongside previously published indexes for 9 of these 11 advanced cancers that were created based on expert provider surveys, also implemented at NCCN Member Institutions. ${ }^{2}$ Final results are 11 symptom indexes that reflect the highest priorities of people affected by these $11 \mathrm{ad}$ - 
vanced cancers and the experienced perspective of the people who provide their medical treatment. Beyond the clinical value of such indexes, they may also contribute significantly to satisfying regulatory requirements for a standardized tool to evaluate drug efficacy with respect to symptomatology.

Over the past 20 years, health-related quality of life (HRQOL) has become an accepted end point in clinical trials. Defined by most as a multidimensional concept that includes self-reported symptoms, functional abilities, and physical, mental, and social health perceptions, HRQOL is measured with a range of valid instruments. ${ }^{3-7}$ Results from HRQOL assessments in oncology research have informed treatment decisions at the group and individual levels. However, oncology health care experts, including regulatory agencies, have voiced concerns about the use of these multi-item, multi-dimensional instruments. ${ }^{1,8}$ Furthermore, physicians have been resistant to implement quality of life assessment in their practices and in many clinical trials. ${ }^{9-11}$ Practical barriers include time and resource constraints, and the perceived lack of a suitable questionnaire. ${ }^{8,12,13}$ Clinical researchers and practicing oncologists acknowledge the absence of a gold standard among HRQOL instruments and confusion about how to interpret HRQOL information, understand the clinical meaningfulness of scores, and translate them into treatment decisions. ${ }^{8,13-19}$

The FDA is also confronted with issues concerning the multidimensional nature and assessment of HRQOL and the consequent implications for claims of drug effectiveness. ${ }^{20}$ In recognizing a need to guard against pharmaceutical manufacturers' "claim expansiveness," in which a promotional claim goes beyond what was measured in the supporting study, ${ }^{1,21}$ the FDA Oncology Drug Advisory Committee subcommittee on Quality of Life has advanced the position that overall claims of HRQOL cannot be made from 1 or 2 domain measurements, and that claims must be specific to the domain that was measured. ${ }^{22-24}$ This committee has also suggested that assessment of symptoms might represent a reasonable starting point in working toward a goal of more focused assessment of HRQOL domains.

\section{Symptom Assessment}

The importance of symptom control in cancer has been widely recognized because of the extraordi- narily high prevalence of physical and psychological symptoms and the impact of these symptoms on HRQOL..$^{25,26}$ For patients with advanced disease, in whom life expectancy is reduced and curative options are limited, relief of physical symptoms and maintenance of function become primary objectives of medical intervention. ${ }^{12,27,28}$ The ability of a new chemotherapeutic agent to ameliorate symptoms specific to a given tumor can represent efficacy in achieving a meaningful patient outcome.

Most validated measures of cancer-specific quality of life incorporate an assessment of certain prevalent symptoms, such as pain and fatigue, within a broader multidimensional assessment.,4,25,26 Cancer-specific HRQOL questionnaires, such as the Functional Assessment of Cancer Therapy-General $\left(\right.$ FACT-G ${ }^{4}$ ) and EORTC QLQ-C30, ${ }^{3}$ assess common cancer symptoms such as pain, fatigue, and nausea in their "core" assessment, then add more detailed, site-specific symptom assessment to that core. One consequence of this measurement approach is that symptoms of most interest to clinicians (and clinician reviewers at the FDA) are embedded in longer multidimensional questionnaires and cannot readily be aggregated into clinically relevant, responsive symptom indexes. A common request, therefore, is a more symptom-focused approach to HRQOL assessment, whereby these multidimensional HRQOL questionnaires aggregate the symptoms being measured in a clinically relevant and psychometrically acceptable manner. Disease-specific measures are more likely to be sensitive to the impact of drug therapy ${ }^{29}$ underscoring the importance of using tumor-specific symptom lists, as opposed to a generic symptom list, to assess drug efficacy across the broad spectrum of tumors.

\section{Groundwork in Symptom Index Development}

Two prerequisites to constructing symptom lists for evaluating response to chemotherapy in advanced cancer are 1) information from patients and 2) input from clinicians on the presence and relative importance of the array of symptoms and concerns associated with a given cancer. This study made significant advancement toward both of these prerequisites. First, procedures used to develop the questions on the FACT-G and its tumor-specific scales required information to be gathered from both cli- 
nicians and patients. Existing FACT item composition was determined using a standardized procedure in which patients and experts, usually in a 3:1 ratio, respectively, were asked to nominate and prioritize important symptoms and concerns of each disease. ${ }^{4,5}$ Second, the previously completed comprehensive survey of physician and nurse experts at $17 \mathrm{NCCN}$ Member Institutions provided detailed information on priority symptoms endorsed by oncology experts for 9 tumor sites. ${ }^{2}$

Results from this expert survey were used to construct 9 tumor-specific indexes of the most important symptoms and concerns to monitor when evaluating treatment for advanced bladder, brain, breast, colorectal, head and neck, hepatobiliary/pancreatic, lung, ovarian, and prostate cancers. This work resulted from a 2-step process. First, a list of symptoms related to cancer in general and each specific tumor was extracted from the FACT and its tumor-specific scales through independent review by 2 medical oncologists and the Principal Investigator of this study (DC), who is a clinical psychologist specializing in HRQOL assessment. Second, these symptoms (plus several others recommended by an independent expert panel) for each of the tumor sites were presented to physicians $(n=223)$ and nurses $(n=232)$ for their selection of the 5 most important symptoms to address in treating patients with these types of cancer. The resulting NCCN/FACT symptom indexes comprised 6 to 15 items, depending on tumor site.

Although the results of this previous work showed good agreement among expert physicians and nurses on the relative importance of symptoms in this survey, ${ }^{2}$ patient ratings of symptom relevance and severity often differ from those of providers, ${ }^{30-34}$ especially regarding psychological symptoms and concerns. ${ }^{34-36}$ The expert survey showed fatigue, pain, nausea, weight loss, and worry to be most frequently endorsed across all 9 indexes, ${ }^{2}$ but patients may have endorsed other symptoms and concerns more (or less) frequently. Although the candidate items presented to experts for selection were drawn from the FACT HRQOL measurement system, and therefore were derived from a list of symptoms identified by patients with that disease as being significant to their HRQOL, the resulting symptom indexes may not have fully reflected the symptom priorities of the patient. In addition, the fact that experts occasionally wrote in items suggested that the instruments contained most, but not all, important disease-related symptoms. Therefore, patients may have viewed as important additional symptoms or concerns that were not on the original list.

The investigators previously showed that oncology experts have similar views about the symptoms that are most important to monitor when treating patients with particular advanced cancers. ${ }^{2}$ They also showed that almost all of the symptoms identified by experts as the most important to assess in treating patients with advanced cancer can be derived from a well-established multidimensional HRQOL questionnaire. They subsequently expanded this work to include a kidney cancer symptom index developed in a similar fashion. ${ }^{37}$ Their existing 10 symptom indexes (9 NCCN-based and 1 FACT-Kidney Symptom Index [FKSI]) provided a starting point for further development of the most important symptom indexes suitable for regulatory review in the context of evaluating treatments for advanced cancer.

\section{Study Design and Overview}

Using the same survey method applied to physicians and nurses, investigators sought patient input to evaluate concordance with symptom priorities and thereby modify the FACT/NCCN indexes accordingly. A symptom index was also developed for lymphoma based on input from patients in this study. Investigators intended to determine the symptoms or concerns that could primarily be attributed to each of the 11 diseases. Ultimately, the appropriate measurement of symptoms judged to be high priority by both clinicians and patients can then be applied to evaluate the effectiveness of noncurative therapy in these diseases.

The primary objective of the current work was to identify patients' highest priority cancer symptoms for 11 advanced cancers, compare their priority ratings with those of oncology experts, and construct brief symptom indexes using combined input (from physicians, nurses, and patients) to assess these symptoms and concerns. Priority symptoms were assessed using surveys of candidate items derived from the well-established FACT-G and 11 FACT tumorspecific scales. Investigators compared patient input with data previously obtained from national and international oncology experts to determine which disease-related symptoms/concerns are most important to monitor in advanced cancers. Patients also had the 
opportunity to add items not present on the FACT$\mathrm{G}$ or disease-specific scales. Items endorsed most frequently by both patients and medical experts were retained on the 11 symptom indexes, and physician input was obtained as to which of these symptoms are considered to be disease- versus treatment-related.

After the survey, patients were asked to complete the FACT HRQOL questionnaire specific to their disease (e.g., FACT-Breast for those with breast cancer). These data allowed initial validation of the symptom indexes through extracting the symptom index items from the full-length HRQOL questionnaire and conducting preliminary analyses of their reliability and validity. Future studies will validate the symptom indexes as standalone scales.

\section{Participants}

The goal was to study 50 patients per disease site. The primary goal was confidence in the stability of the individual item rankings, which is why 50 was set as the target. A secondary goal was to ensure enough patients were included to achieve saturation of information with regard to new content to add beyond the FACT-G items. Because prior research has tended to find saturation of content with 15 to 25 respondents, the investigators considered 50 to be a conservative target. Patients were eligible for the study if they were at least 18 years of age and had stage III or IV bladder, brain, breast, colorectal, head and neck, hepatobiliary/pancreatic, kidney, lung, ovarian, or prostate cancer or lymphoma. Patients must have had prior experience with chemotherapy for at least 2 cycles (or 1 month for noncyclical chemotherapy), and no other primary malignancy diagnosed or treated within the previous 5 years, excluding nonmelanoma skin cancer. A total of 533 patients participated in the study, with approximately 50 patients per disease site except for bladder cancer $(\mathrm{n}=31)$. Table 1 displays demographic and clinical information for the sample. Physicians were eligible to complete the disease-/treatment-related survey if they were currently in practice at any of the 21 NCCN Member Institutions and had at least 3 years' experience treating a minimum of 100 patients with 1 of the 11 target diseases. A total of 91 oncologists from 18 NCCN Member Institutions completed 112 surveys.

\begin{tabular}{|c|c|c|}
\hline & $\mathbf{n}$ & Percentage (\%) \\
\hline Female & 257 & 48.2 \\
\hline Hispanic & 16 & 3.0 \\
\hline \multicolumn{3}{|l|}{ Race/Ethnicity } \\
\hline White & 474 & 89.1 \\
\hline African-American & 44 & 8.3 \\
\hline Asian & 8 & 1.5 \\
\hline Other & 6 & 1.1 \\
\hline \multicolumn{3}{|l|}{ Cancer Diagnosis } \\
\hline Bladder & 31 & 5.8 \\
\hline Brain & 50 & 9.4 \\
\hline Breast & 52 & 9.7 \\
\hline Colorectal & 50 & 9.4 \\
\hline Head and neck & 49 & 9.2 \\
\hline Hepatobiliary & 50 & 9.4 \\
\hline Kidney & 50 & 9.4 \\
\hline Lung & 50 & 9.4 \\
\hline Lymphoma & 50 & 9.4 \\
\hline Ovarian & 51 & 9.6 \\
\hline Prostate & 50 & 9.4 \\
\hline \multicolumn{3}{|c|}{ Patient-Rated ECOG Performance Status Rating } \\
\hline 0, normal activity & 122 & 22.8 \\
\hline 1, some symptoms & 258 & 48.3 \\
\hline $2,<50 \%$ bedrest & 133 & 24.9 \\
\hline $3,>50 \%$ bedrest & 20 & 3.8 \\
\hline
\end{tabular}

Mean age, 59 years (standard deviation, 11.9); median age, 59 years (range, $24-88$ years)

Abbreviation: SD, standard deviation.

\section{Study Procedures}

Patients were recruited from 2 sources: 1) $5 \mathrm{NCCN}$ Member Institutions, namely Dana-Farber Cancer Institute, Duke University Medical Center, Fred Hutchinson Cancer Research Center, H. Lee Moffitt Cancer Center \& Research Institute, and Robert $\mathrm{H}$. Lurie Comprehensive Cancer Center of Northwestern University; and 2) the Cancer Health Alliance of Metropolitan Chicago (CHAMC), a coalition of 4 community support agencies serving the Chicago metropolitan area. These organizations provide free social, emotional, and informational support services to patients with cancer, and are unaffiliated with a medical center or university. They were selected to obtain patient input from community-based practices to balance 
the preferences of patients from tertiary referral centers such as NCCN sites.

Patients were first asked to generate up to $10 \mathrm{im}$ portant symptoms or concerns to monitor when assessing the value of chemotherapy for their disease, and then asked to rank the importance of each on a scale of 0 to 10 . Patients then filled out a checklist on which they selected 5 top symptoms/concerns from among 23 to 45 items included in the FACT HRQOL questionnaire or nominated by clinician experts in the particular cancer type. The checklists administered to patients in this study were identical to those administered to NCCN physicians and nurses in the investigators' previous study. ${ }^{2}$ Four versions of each checklist were created to control for response bias because of order effect. On the checklist, patients were first asked to select no more than 10 symptoms or concerns that they believed were "the most important symptoms or concerns to monitor when assessing the value of drug treatment for advanced <site> cancer." Of the 10 symptoms/concerns nominated as "the most important," patients were then asked to select up to 5 as "the very most important." Space was provided for respondents to write in symptoms or concerns that were not already listed. Patients then completed the site-specific FACT HRQOL measure containing approximately 35 to 50 questions on patients' physical, functional, social/family, and emotional well-being and additional cancer-specific topics.

Physicians from all 21 NCCN Member Institutions were recruited via e-mail by NCCN headquarters staff. On the physician survey, completed online using SurveyMonkey, respondents were asked to rate each symptom/concern on a 5-point scale as to whether it was "exclusively disease-related", "predominantly disease-related", "too close to determine", "predominantly a treatment side effect," or "exclusively a treatment side effect." Another category allowed physicians to indicate if they believed the symptom was neither disease- nor treatment-related.

\section{Analysis Plan}

Patient surveys were tabulated according to the frequency with which they selected a particular symptom-concern as 1 of the 5 most important. By chance, the probability of item endorsement as one of the top 5 symptoms is calculated by dividing 5 (the allowable number of "very most important symptoms") by the total number of items in each disease-specific checklist. Items whose patient ratings exceeded that probability were retained for consideration of inclusion in the final index. These retained items, endorsed by patients for each of the 11 tumor sites, were then compared with expert clinician responses obtained in the investigators' previous work. Expert clinician and patient ratings were given equal weight in item selection. If patients endorsed a symptom/concern substantially more than others, then that symptom/ concern was reviewed for inclusion of a second item targeting an aspect of that symptom experience in the index. Where possible, items to be added were extracted from the Functional Assessment of Chronic Illness Therapy (FACIT) measurement system for ease of use, validity, and translation capabilities.

Write-in items were also reviewed for existence of new concepts that had not previously appeared on the checklists. If greater than $10 \%$ of patients in a disease category reported a particular new symptom/ concern that was not judged to be treatment-related (by the Principal Investigator), or greater than 20\% of patients if the new symptom/concern was judged to be primarily treatment-related, it was considered for item inclusion. Additionally, if the symptom/ concern was highly endorsed on the checklists and was also highly reported in the patient-generated lists, that symptom/concern was reviewed for inclusion of a second item in the index.

Physician surveys were tabulated for classification of each index item into Disease-Related Symptom (DRS), Treatment Side Effect (TSE), and general Function and Well-Being (FWB) subscales. Index items were reviewed for classification as "exclusively/predominantly disease-related," "exclusively/predominantly treatment-related," or "too close to determine/neither." If more than $50 \%$ of physicians in a disease category indicated an item was exclusively/predominantly treatment-related, these items were categorized in the TSE subscale. The remainder of items were determined to be either exclusively/predominantly disease-related (resulting in membership on the DRS subscale) or descriptive of general functioning (FWB subscale).

\section{Results}

Table 2 displays the number of symptoms presented and the number endorsed with a probability greater than chance. Table 3 displays the frequency of items endorsed 


\begin{tabular}{|c|c|c|}
\hline Cancer Type & $\begin{array}{l}\text { Number of } \\
\text { Symptoms } \\
\text { or } \\
\text { Concerns } \\
\text { Presented } \\
\text { on Survey* }\end{array}$ & $\begin{array}{l}\text { Minimum Number } \\
\text { of Patients Needed } \\
\text { to Select an Item in } \\
\text { Their "Top 5" to be } \\
\text { Considered Greater } \\
\text { Than Chance }^{\dagger}\end{array}$ \\
\hline Bladder & 28 & 6 \\
\hline Brain & 40 & 7 \\
\hline Breast & 23 & 12 \\
\hline Colorectal & 26 & 10 \\
\hline Head and neck & 28 & 9 \\
\hline Hepatobiliary & 35 & 8 \\
\hline Kidney & 26 & 10 \\
\hline Lung & 28 & 9 \\
\hline Lymphoma & 39 & 7 \\
\hline Ovarian & 30 & 9 \\
\hline Prostate & 31 & 9 \\
\hline
\end{tabular}

*Three items were added to each checklist partway through the study.

${ }^{+}$Number of patients needed is the first whole number larger than the product of 5 divided by the number of items on the checklist multiplied by the number of patients.

by patients for the total sample by disease, including the most frequent patient-rated "Top 5" symptoms and concerns across each of the 11 cancer types.

Symptoms/concerns were reviewed for inclusion of a second index item if patients endorsed it substantially more than others. The most common example of this was the addition of "I feel fatigued" to "I have a lack of energy" on disease-specific indexes because many more patients rated this symptom in their top 5 compared with other symptoms. Nearly all items to be added were extracted from the FACIT measurement system for ease of use, validity, and translation capabilities. Some disease groups had a high percentage of patients (e.g., 30\% of the breast cancer sample) reporting medication or treatment side-effects as one of the most important symptoms to monitor. Therefore, the item "I am bothered by side effects of treatment" from the FACIT system was added to all indexes.

After $30 \%(n=107)$ of the patient-generated lists and $51 \%(n=275)$ of the patient checklists were collected, an interim analysis of patient-generated symptoms and/or concerns was conducted to determine whether any new concepts or items should be added to the physician survey. Several disease groups identified 11 new symptoms/concerns, although some of these "new" symptoms already appeared on other disease checklists. As a result, 7 to 9 items were added to each disease-specific physician survey for diseaseor treatment-related classification. To ensure saturation of information regarding new item content, the investigators checked and confirmed that all new item content included in the indexes had been identified within the first 30 patients per disease site.

The resulting NCCN/FACT symptom indexes comprise 16 to 24 items, depending on tumor site. Symptoms and concerns receiving the most consistent endorsement across the disease groups included lack of energy (fatigue), ability to enjoy life, worry condition will worsen, nausea, ability to sleep well, contentment with QOL, and pain. Table 4 displays descriptive statistics for the new NCCN/FACT index scores.

\section{Discussion and Future Directions}

This article summarizes an effort to reconfigure, and augment as needed, questions in the FACT Measurement System that address the most important symptoms and concerns of people with 11 different types of cancer. The investigators identified patients' highest-priority cancer symptoms across 11 advanced cancers, and then compared those priorities with those of oncology experts. This information was then used to construct brief symptom indexes using combined input (i.e., physicians, nurses, patients) to assess these symptoms and concerns. Priority symptoms were assessed using open-ended qualitative input from patients and surveys of candidate items derived from the FACT-G and 11 FACT tumorspecific scales. The investigators compared patient input with data already obtained from national and international oncology experts to determine which symptoms/concerns were most important to monitor in advanced cancers. Items endorsed most frequently by both patients and medical experts were retained on the 11 new symptom indexes for advanced bladder, brain, breast, colorectal, head and neck, hepatobiliary, kidney, lung, ovarian, and prostate cancers and lymphoma. Indexes have been formatted by subscale, separating DRS, TSE, and general FWB items for ease of use and scoring.

The reconfiguration of questions into concise symptom indexes accomplishes several objectives, including brevity, clinical relevance, and content 
Cella et al.

Table 3 Patient Rankings of Symptoms/Concerns by Cancer Type (cont.)

\begin{tabular}{|c|c|c|c|}
\hline Cancer Type & Rank & \% Endorsed (Top 5) & Symptom or Concern \\
\hline \multirow[t]{9}{*}{ Bladder } & 1 & 48 & Lack of energy (fatigue) \\
\hline & 2 & 45 & Worry condition will get worse \\
\hline & 3 & 32 & Trouble meeting needs of family \\
\hline & 4 & 26 & Able to enjoy life \\
\hline & 4 & 26 & Control of bowels \\
\hline & 6 & 23 & Having and maintaining an erection \\
\hline & 6 & 23 & Content with quality of life \\
\hline & 6 & 23 & Nausea \\
\hline & 9 & 19 & Able to sleep well \\
\hline \multirow[t]{12}{*}{ Brain } & 1 & 44 & Lack of energy (fatigue) \\
\hline & 2 & 22 & Frustrated can't do usual things \\
\hline & 3 & 20 & Able to enjoy life \\
\hline & 4 & 18 & Nausea \\
\hline & 4 & 18 & Seizures (convulsions) \\
\hline & 4 & 18 & Trouble with coordination \\
\hline & 4 & 18 & Worry condition will get worse \\
\hline & 8 & 16 & Change in personality \\
\hline & 8 & 16 & Able to find right word(s) \\
\hline & 8 & 16 & Able to remember new things \\
\hline & 8 & 16 & Worry about dying \\
\hline & 12 & 14 & Able to concentrate \\
\hline \multirow[t]{8}{*}{ Breast } & 1 & 58 & Lack of energy (fatigue) \\
\hline & 2 & 33 & Able to enjoy life \\
\hline & 3 & 31 & Able to work \\
\hline & 3 & 31 & Worry condition will get worse \\
\hline & 5 & 25 & Pain \\
\hline & 6 & 23 & Able to sleep well \\
\hline & 6 & 23 & Significant pain (bone pain) \\
\hline & 6 & 23 & Feeling ill \\
\hline \multirow[t]{6}{*}{ Colorectal } & 1 & 62 & Lack of energy (fatigue) \\
\hline & 2 & 30 & Able to enjoy life \\
\hline & 3 & 28 & Content with quality of life \\
\hline & 3 & 28 & Nausea \\
\hline & 5 & 26 & Control of bowels \\
\hline & 6 & 22 & Diarrhea \\
\hline \multirow{12}{*}{ Head and neck } & 1 & 39 & Pain in mouth, throat, and neck \\
\hline & 1 & 39 & Able to swallow \\
\hline & 3 & 35 & Lack of energy (fatigue) \\
\hline & 4 & 31 & Nausea \\
\hline & 5 & 22 & Able to eat foods I like \\
\hline & 5 & 22 & Able to enjoy life \\
\hline & 5 & 22 & Able to sleep well \\
\hline & 5 & 22 & Feeling ill \\
\hline & 9 & 20 & Trouble meeting needs of family \\
\hline & 10 & 18 & Able to eat solid foods \\
\hline & 10 & 18 & Able to work \\
\hline & 10 & 18 & Weight loss \\
\hline \multirow[t]{12}{*}{ Hepatobiliary } & 1 & 36 & Lack of energy (fatigue) \\
\hline & 2 & 30 & Worry condition will get worse \\
\hline & 3 & 26 & Able to enjoy life \\
\hline & 3 & 26 & Fatigue \\
\hline & 3 & 26 & Nausea \\
\hline & 6 & 24 & Able to do usual activities \\
\hline & 7 & 22 & Good appetite \\
\hline & 7 & 22 & Pain \\
\hline & 9 & 20 & Discomfort or pain in stomach area \\
\hline & 10 & 18 & Worry about dying \\
\hline & 10 & 18 & Able to sleep well \\
\hline & 10 & 18 & Content with quality of life \\
\hline
\end{tabular}


Advanced Cancer Index

\section{Table 3 Patient Rankings of Symptoms/Concerns by Cancer Type (cont.)}

\begin{tabular}{|c|c|c|c|}
\hline Cancer Type & Rank & \% Endorsed (Top 5) & Symptom or Concern \\
\hline \multirow[t]{7}{*}{ Kidney } & 1 & 52 & Side effects of treatment \\
\hline & 2 & 42 & Lack of energy (fatigue) \\
\hline & 3 & 34 & Fatigue \\
\hline & 4 & 30 & Worry condition will get worse \\
\hline & 5 & 28 & Able to enjoy life \\
\hline & 6 & 20 & Able to work \\
\hline & 6 & 20 & Pain \\
\hline \multirow[t]{11}{*}{ Lung } & 1 & 44 & Lack of energy (fatigue) \\
\hline & 2 & 30 & Able to enjoy life \\
\hline & 2 & 30 & Worry condition will get worse \\
\hline & 4 & 26 & Able to sleep well \\
\hline & 4 & 26 & Nausea \\
\hline & 4 & 26 & Shortness of breath \\
\hline & 7 & 24 & Good appetite \\
\hline & 7 & 24 & Able to think clearly \\
\hline & 9 & 22 & Able to breathe easily \\
\hline & 10 & 18 & Able to enjoy usual activities \\
\hline & 10 & 18 & Significant pain (bone pain) \\
\hline \multirow[t]{13}{*}{ Lymphoma } & 1 & 38 & Lack of energy (fatigue) \\
\hline & 2 & 34 & Getting tired easily \\
\hline & 3 & 28 & Able to enjoy life \\
\hline & 4 & 26 & Worry about getting infections \\
\hline & 5 & 22 & Trouble concentrating \\
\hline & 6 & 20 & Lumps or swelling \\
\hline & 6 & 20 & Nausea \\
\hline & 6 & 20 & Worry condition will get worse \\
\hline & 9 & 18 & Able to support self or family \\
\hline & 9 & 18 & Uncertain about future health \\
\hline & 11 & 16 & Difficulty planning for future \\
\hline & 11 & 16 & Content with quality of life \\
\hline & 11 & 16 & Emotional ups and downs \\
\hline \multirow[t]{10}{*}{ Ovarian } & 1 & 61 & Lack of energy (fatigue) \\
\hline & 2 & 37 & Able to sleep well \\
\hline & 3 & 25 & Able to enjoy life \\
\hline & 4 & 24 & Content with quality of life \\
\hline & 5 & 22 & Able to get around \\
\hline & 5 & 22 & Constipation \\
\hline & 5 & 22 & Nausea \\
\hline & 8 & 20 & Pain \\
\hline & 8 & 20 & Worry condition will get worse \\
\hline & 10 & 18 & Feeling ill \\
\hline \multirow[t]{7}{*}{ Prostate } & 1 & 56 & Lack of energy (fatigue) \\
\hline & 2 & 32 & Worry condition will get worse \\
\hline & 3 & 24 & Able to enjoy life \\
\hline & 4 & 20 & Good appetite \\
\hline & 4 & 20 & Content with quality of life \\
\hline & 6 & 18 & Satisfaction with sex life \\
\hline & 6 & 18 & Weakness in legs \\
\hline
\end{tabular}


validity given their link to the range of patient-based values. By focusing on patient-driven priorities, these indexes reflect the most important symptoms and concerns faced by people who are undergoing treatment for advanced cancer. The inclusion of input from expert providers regarding priorities, and the extent to which a concern can be regarded as influenced by disease versus treatment, enables the investigators to consider dividing these concise indexes into 3 compartments: DRS, TSE, and general FWB. The hope is that these symptom indexes will address expressed concerns of regulatory agencies, oncology health experts, physicians, and patients being treated for advanced cancer. Because of their brevity and focus on relevant symptoms and concerns, applying these indexes in clinical practice evaluation may also be useful. Given that each index is fewer than 25 items in length (range, 16-24), they may help address provider concerns regarding patient burden, impact of assessment on practice throughput, time and recourse constrains, and interpretation of results. Because they are focused on patients' most important concerns, but not all concerns, their use in a regulatory environment may help protect against "claim expansiveness" that has historically been noted. Further research in these areas would be of particular value.

The items presented to patients for selection were drawn from the FACIT Measurement System, which in turn were derived from a list of symptoms and concerns originally identified by patients with that disease. Because patients were allowed to write in symptoms/concerns that may not have appeared on the symptom checklists, inclusiveness of the original FACT scale can be gauged. Most of the symptoms and concerns patients believe are important to monitor when receiving treatment for these advanced cancers were included. Based on this, 2 lines of research going forward would be of particular value. First, validation of these targeted indexes which, although approximately $90 \%$ drawn from FACT scales, do include $10 \%$ new content, is important in longitudinal clinical research. Although the FACT scales have been in use and have associated benchmark ("normative") data and empiric standards for meaningful difference and change, these standards must be established for the indexes. A second line of important research is in the clinical usefulness of routine monitoring of these important concerns with regard to their potential impact on decision-making, quality of care, and HRQOL.

The methodology used in this investigation has both strengths and weaknesses. It is highly transparent, with patient ratings of importance summed to form indexes comprising the most frequently identified concerns. Another strength is the parallel inclusion of expert rankings and attributions as to whether the most important symptoms or concerns are primarily attributable to disease or treatment, providing clinically relevant labels to item subsets

\section{Table 4 Descriptive Statistics for NCCN/FACT Symptom Index Scores}

\begin{tabular}{|lllll|}
\hline Cancer Type & $\begin{array}{l}\text { Number of Items on } \\
\text { Index (New Items*) }\end{array}$ & $\begin{array}{l}\text { Possible Range } \\
\text { of Index Scores }\end{array}$ & Mean Index Score (SD) & Mean FACT-G (SD) \\
\hline Bladder & $18(2)$ & $0-72$ & $46.3(11.4)$ & $75.6(14.5)$ \\
Brain & $24(3)$ & $0-96$ & $67.3(13.6)$ & $78.8(13.5)$ \\
Breast & $16(3)$ & $0-64$ & $42.3(12.1)$ & $76.9(18.0)$ \\
Colorectal & $19(4)$ & $0-76$ & $55.3(11.7)$ & $79.5(13.7)$ \\
Head and neck & $22(4)$ & $0-88$ & $53.1(14.5)$ & $74.6(14.7)$ \\
Hepatobiliary & $18(0)$ & $0-72$ & $45.7(12.8)$ & $72.7(16.8)$ \\
Kidney & $19(2)$ & $0-76$ & $49.4(11.5)$ & $78.5(14.8)$ \\
Lung & $17(2)$ & $0-68$ & $46.1(9.1)$ & $78.2(13.9)$ \\
Lymphoma & $18(2)$ & $0-72$ & $48.8(12.0)$ & $81.8(16.0)$ \\
Ovarian & $18(2)$ & $0-72$ & $51.6(10.7)$ & $78.1(15.1)$ \\
Prostate & $17(2)$ & $0-68$ & $46.2(8.8)$ & $82.0(13.5)$ \\
\hline
\end{tabular}

Abbreviations: FACT, Functional Assessment of Cancer Therapy; G, general; SD, standard deviation.

*The number of items added to the index based on patient feedback that were not administered as part of a FACT questionnaire. Index scores have been prorated upward to account for this missing data. 
of each index. A weakness of this method includes vulnerability to sampling bias among patients; a weakness the investigators attempted to overcome by including approximately 50 patients per disease site, which is 2 to 3 times more than typically included in similar qualitative studies. These indexes can be regarded as possessing very good content validity; patients were encouraged to supplement items drawn from the FACT with new content, and these new items were then added to the indexes. This approach enhances content validity, but disabled the ability to evaluate internal consistency, because the new (added) items were not included in the index tested with patients. Further evaluation of reliability and validity, including responsiveness to change, is therefore recommended.

These indexes are targeted to patients with stage III or IV cancer who are undergoing chemotherapy. Although this is a fairly narrow group, differences may exist regarding the amount or type of prior chemotherapy. In some diseases, symptoms may vary according to stage or location of metastases. Further evaluation of the usefulness of these indexes across different disease and treatment subgroups would be useful. In addition, patient valuation of symptoms may be related to characteristics such as education, socioeconomic status, sex, age, culture, or race/ethnicity. To evaluate this, future research can explore these factors as they relate to symptom valuation and the intensity of the concerns noted.

Measurement of the high-priority symptoms captured in these indexes can be applied to evaluate the effectiveness of therapy in these 11 diseases. Developed using methods that meet industry standards for ensuring content validity and that are consistent with recent guidance from the FDA,${ }^{1}$ these symptom indexes may be helpful in regulatory submission when evaluating the impact of new treatments on the symptoms that matter most to people with advanced cancer. Regulatory review will ultimately determine their acceptability for approved label claims, and will undoubtedly shape research into the future. Further evaluation of their usefulness in clinical research and clinical practice is anticipated.

\section{Acknowledgments}

Portions of these findings were presented at the 2007 ASCO Annual Meeting. The study from which the results were drawn was designed and conducted by independent investigators at Evanston Northwestern Healthcare and NCCN.

\section{References}

1. Guidance for Industry: patient-reported outcome measures: use in medical product development to support labeling claims. U.S. Food and Drug Administration Web site. Available at: http://www.fda.gov/ downloads/Drugs/GuidanceComplianceRegulatoryInformation/ Guidances/UCM193282.pdf. Accessed January 20, 2011.

2. Cella DF, Paul D, Yount $S$, et al. What are the most important symptom targets when treating advanced cancer? A survey of providers in the National Comprehensive Cancer Network (NCCN). Cancer Invest 2003;21:526-535.

3. Aaronson NK, Ahmedzai S, Bergman B, et al. The European Organization for Research and Treatment of Cancer QLQ-C30: a quality-of-life instrument for use in international clinical trials in oncology. J Natl Cancer Inst 1993;85:365-376.

4. Cella DF, Tulsky DS, Gray G, et al. The Functional Assessment of Cancer Therapy (FACT) Scale: development and validation of the general measure. J Clin Oncol 1993;11:570-579.

5. Cella DF, Bonomi AE. Measuring quality of life: 1995 update. Oncology 1995;9(Suppl):47-60.

6. Schag CA, Heinrich RL. Development of a comprehensive quality of life measurement tool: CARES. Oncology (Williston Park) 1990;4:135-138.

7. Schipper H, Clinch J, McMurray A, et al. Measuring the quality of life of cancer patients: the Functional Living Index-Cancer: development and validation. J Clin Oncol 1984;2:472-483.

8. Taylor KM, Macdonald KG, Bezak A, et al. Physicians' perspective on quality of life: an exploratory study of oncologists. Qual Life Res 1996;5:5-14.

9. Bezjak A, Taylor KM, Ng P, et al. Quality-of-life information and clinical practice: the oncologist's perspective. Cancer Prev Control 1998;2:230-235.

10. Deyo RA, Patrick DL. Barriers to the use of health status measures in clinical investigation, patient care, and policy research. Med Care 1989;27(Suppl):S254-268.

11. Nelson EC, Berwick DM. The measurement of health status in clinical practice. Med Care 1989;27(Suppl):S77-90.

12. Fish LS, Lewis BE. Quality of life issues in the management of ovarian cancer. Semin Oncol 1999;26(Suppl 1):32-39.

13. Morris J, Perez D, McNoe B. The use of quality of life data in clinical practice. Qual Life Res 1998;7:85-91.

14. Browman GP. Science, language, intuition, and the many meanings of quality of life. J Clin Oncol 1999;17:1651-1653.

15. Gill TM, Feinstein AR. A critical appraisal of the quality of quality-of-life measurements. JAMA 1994;272:619-626.

16. Guyatt G, Feeny DH, Patrick DL. Measuring health-related quality of life. Ann Intern Med 1993;118:622-629.

17. Hopwood P. Progress, problems, and priorities in quality of life research. Eur J Cancer 1992;28A:1748-1752.

18. Skeel R. Quality of life dimensions that are most important to cancer patients. Oncology 1993;7:55-70.

19. Wilson IB, Cleary PD. Linking clinical variables with healthrelated quality of life: a conceptual model of patient outcomes. JAMA 1995;273:59-65. 


\section{Cella et al.}

20. Rothermich EA, Pathak DS. References for health-related quality of life claims in prescription drug advertisements. Am J Health Syst Pharm 1997;43:2596-2599.

21. F-D-C Report: "The Pink Sheet.". Quality of life ad claims should be specific, FDA-sponsored physician focus groups say: ad division direction cautions against QOL “claim expansiveness." 1994;56:89.

22. Beitz J. Quality-of-life end points in oncology drug trials. Oncology 1999;13:1439-1447.

23. Quality Of Life Subcommittee of the Oncologic Drugs Advisory Committee, February 10, 2000. U.S. Food and Drug Administration Web site. Available at: http://www.fda.gov/ohrms/dockets/ac/00/ slides/3591s1.htm. Accessed January 20, 2011.

24. Leidy NK, Revicki DA, Genesté B. Recommendations for evaluating the validity of quality of life claims for labeling and promotion. Value Health 1999;2:113-127.

25. Portenoy RK, Thaler HT, Kornblith AB, et al. Symptom prevalence, characteristics and distress in a cancer population. Qual Life Res 1994a;3:183-189.

26. Portenoy RK, Thaler HT, Kornblith AB, et al. The Memorial Symptom Assessment Scale: an instrument for the evaluation of symptom prevalence, characteristics and distress. Eur J Cancer 1994b;30A:1326-1336.

27. Coons SJ, Kaplan RM. Assessing health-related quality of life: application to drug therapy. Clin Ther 1992;14:850-858.

28. Sutherland HJ, Lockwood GA, Boyd NF. Ratings of the importance of quality of life variables: therapeutic implications for patients with metastatic breast cancer. J Clin Epidemiol 1990;43:661-666.

29. Kong S, Gandhi SK. Methodologic assessments of quality of life measures in clinical trials. Ann Pharmacother 1997;31:830-836.

30. Brunelli C, Costantini M, Di Giulio P, et al. Quality of life evaluation: when do terminal cancer patients and health-care providers agree? J Pain Symptom Manage 1998;15:151-158.

31. Justice AC, Rabeneck L, Hays RD, et al. Sensitivity, specificity, reliability, and clinical validity of provider-reported symptoms: a comparison with self-reported symptoms. J AIDS 1999;21:126133.

32. Nekolaichuk C, Bruera E, Spachynski K, et al. A comparison of patient and proxy symptom assessments in advanced cancer patients. Palliat Med 1999;13:311-323.

33. Stephens RJ, Hopwood P, Girling DJ, et al. Randomized trials with quality of life endpoints: are doctors' ratings of patients' physical symptoms interchangeable with patients' self-ratings? Qual Life Res 1997;6:225-236.

34. Stromgren AS, Groenvold M, Sorensen A, et al. Symptom recognition in advanced cancer: a comparison of nursing records against patient self-rating. Acta Anaesthesiol 2001;45:1080-1085.

35. Lampic C, Nordin K, Sjoden P. Agreement between cancer patients and their physicians in the assessment of patient anxiety at followup visits. Psychooncology 1995;4:301-310.

36. Passik SD, Dugan W, McDonald MV, et al. Oncologists' recognitions of depression in their patients with cancer. J Clin Oncol 1998;16:1594-1600.

37. Rao D, Butt Z, Rosenbloom S, et al. A comparison of the Renal Cell Carcinoma Symptom Index (RCC-SI) and the Functional Assessment of Cancer Therapy-Kidney Symptom Index (FKSI). J Pain Symptom Manage 2009;38:291-298. 